\title{
A NEW ANT OF THE GENUS EPITRITUS FROM SOUTH OF THE SAHARA*
}

\author{
By William L. Brown, Jr. \\ Department of Entomology, Cornell University
}

Recently Mr. G. E. J. Nixon, of the Commonwealth Institute of Entomology in London, sent me a small series of a curious ant that he had recognized as an aberrant and possibly undescribed species of tribe Dacetini. Upon receiving the specimens, I found that the sample represented a new Epitritus, the first member of the genus from Ethiopian Africa, and the first to be found in the tropics. I am grateful to Mr. Nixon for making available this most interesting new species.

\section{Epitritus laticeps sp. nov.}

Figures I-4

Holotype worker: TL 2.2, HL 0.47, HW 0.58 (CI 123), ML 0.22 ( MI 47), WL 0.49, scape L 0.29 , funiculus $L$ o.43 mm, of which about half is taken up by the apical segment. Measurements and proportions are those standard in my other dacetine studies (see Brown, 1953, Amer. Midl. Nat. 50: cf. pp. 7-I 5).

Shape of head, body and mandibles as shown in Figs. I-4. Especially characteristic of the known species of Epitritus and (judging from two occipital lobes (which make the head distinctly broader than long); the wide, basally lobiform antennal scapes; the large, conical labral lobes; the 4 long straplike clypeal hairs and the peculiar mandibles, lacking a long, spiniform dorsal "apical" tooth and with only a single preapical tooth. The apex of the mandible is of the "inverted" type characteristic of the known species of Epitritus and (judging from two paratype workers of which the mandibles were opened) has 7 or 8 denticles, of which one or two in the middle are round-edged, and the rest are acute. The basal lamella is small, with acutely rounded apex (Fig. 3), and the ventro-medial margin below it is obtusely denticulate or angulate (not shown in figures). Eyes minute, with only 4-6 facets. Antennal funiculus clearly 5-segmented.

Promesonotum seen from above broadly oval, almost circular, narrowest behind, where it is terminated by the fine but distinct metanotal groove, which crosses just behind the highest point of the swollen mesonotum. Propodeum much narrower than pronotum, with high,

\footnotetext{
*Manuscript received by the editor December 15, 1961.
} 
angular lamellae guarding the concave declivity, the declivity reaching far up towards the metanotal groove.

Petiolar node distinct from its anterior peduncle, as seen from above transverse, subrectangular, about twice as broad as long. Postpetiole

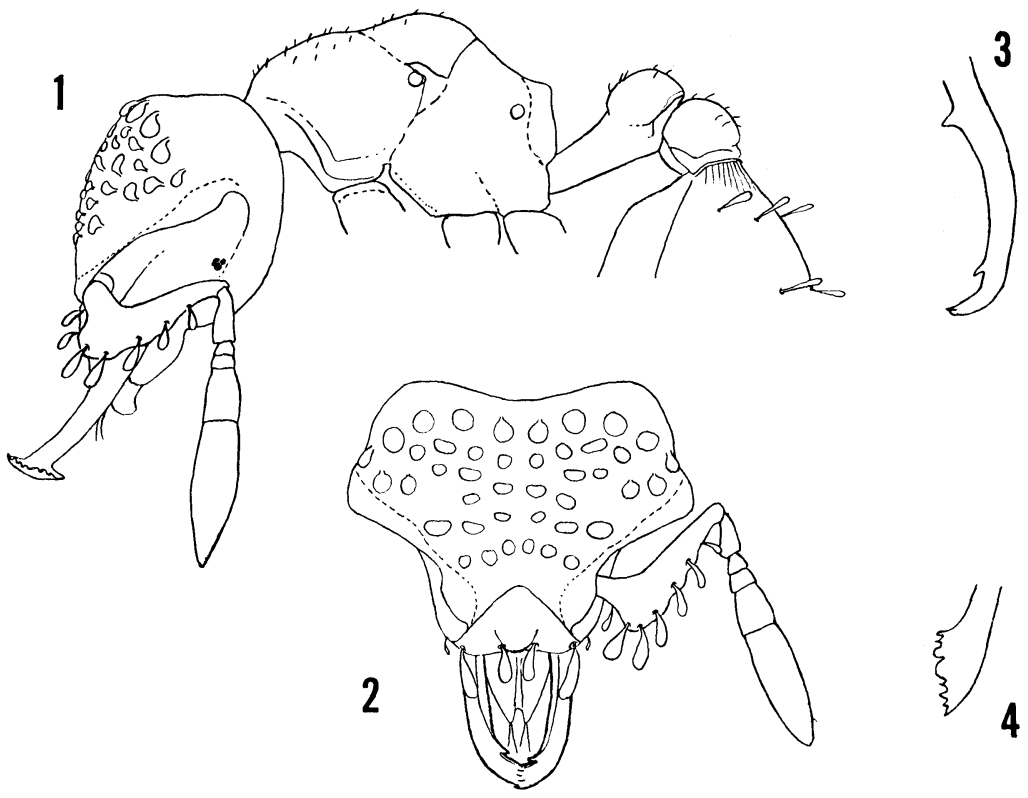

Figures 1-4. Epitritus laticeps sp. nov., paratype worker. Fig. 1, side view of head and body. Fig. 2, dorsal full-face view of head and mandibles. Fig. 3 , oblique dorsal view of opened left mandible. Fig. 4, ventral inside oblique view of apical group of teeth or denticles. Drawn by F. A. McKittrick.

much broader than petiole and more than twice as broad as long, convex above with a shallow median impression, articulated to the entire width of the anterior gastric margin. Spongiform appendages limited to narrow posterodorsal lamelliform collars on both nodes, and absent from anteroventral face of gaster. Gaster laterally submarginate at the extreme base; short, coarse costulae fade caudad into superficial, indistinct reticulation reaching nearly to the midlength of the basal segment; gaster otherwise shining and smooth.

Body reticulate-punctulatc, opaque, except for the gaster, the inner mandibular surfaces and the labral lobes, which are smooth and shining. Specialized pilosity, especially the large orbicular, cochlear hairs 
of the head, and the spatulate ones of the scapes and clypeus, as shown in the figures. Slender, erect spatulate-to-clavate hairs in rows of 4 or 6 on gastric dorsum, totalling about 32. Ground pilosity reduced to minute stubby hairs, chiefly on promesonotum and nodes, and sparse, short reclinate hairs on gastric dorsum and legs. Tibiae and tarsi with longer reclinate hairs, some of them spatulate. Gula with fine reclinate hairs. Color medium ferruginous; head feebly infuscated dorsally; appendages more yellowish.

Holotype [British Museum (Natural History)] and six paratype workers [deposited with holotype and in Museum of Comparative Zoology at Harvard College] taken in northern Nigeria near Zungeru on the Kaduna Road, I9 December, I956, from "base of dead tree" by W. A. Sands of the Termite Research Unit (Collection No. S $780)$. The paratypes are quite similar to the holotype, and range from the same size down to the smallest specimen: TL 2.0, HL 0.46, HW 0.55 (CI I 20), ML 0.22 (MI 48), WL $0.49 \mathrm{~mm}$. Cephalic index range for the entire type series is II9-I25. There is slight variation in the size and shape of the small teeth or denticles in the apical complex. Female and male unknown.

$E$. laticeps can be separated from its two congeners by means of the following key:

I. Tooth at apparent (dorsal) apex of mandible small, about equal to or slightly longer than the other largest teeth of the apical group; promesonotum without conspicuous hairs of any kind (Nigeria) laticeps Brown

Tooth at apparent (dorsal) apex of mandible long, straight and spiniform, about 2 or 3 or more times as long as the largest of the other teeth in the apical group; promesonotum with numerous large orbicular scale-like hairs like those of the dorsum of the head

2. Funiculus with 5 separate segments; mandible with two preapical teeth (Japan: Kyushu, Honshu) ....... hexamerus Brown Funiculus with only 3 separate segments; mandible with 4 preapical teeth (Mediterranean lands n. to Hungary)

argiolus Emery

The finding of a species of Epitritus south of the Sahara marks the genus as a zoogeographical curiosity of more than usual interest. Of the two previously known species, $E$. argiolus is widespread in southern Europe and North Africa, while E. hexameris has been taken twice in Japan. Evidently, Epitritus is a relict-distributed group that was once more widely distributed in the tropical and warm trmperate 
parts of the Old World. The insects are cryptobiotic in habits, and are small and inconspicuous, so we may look forward to the discovery of species elsewhere in the Old World.

Another interesting thing about the new species is its close convergence in many morphological details to Talaridris mandibularis Weber (tribe Basicerotini) of Trinidad and British Guiana (see Brown and Kempf, 196o, Stud. Ent., Petropolis, (n.s.) 3:233, 24I-242). 

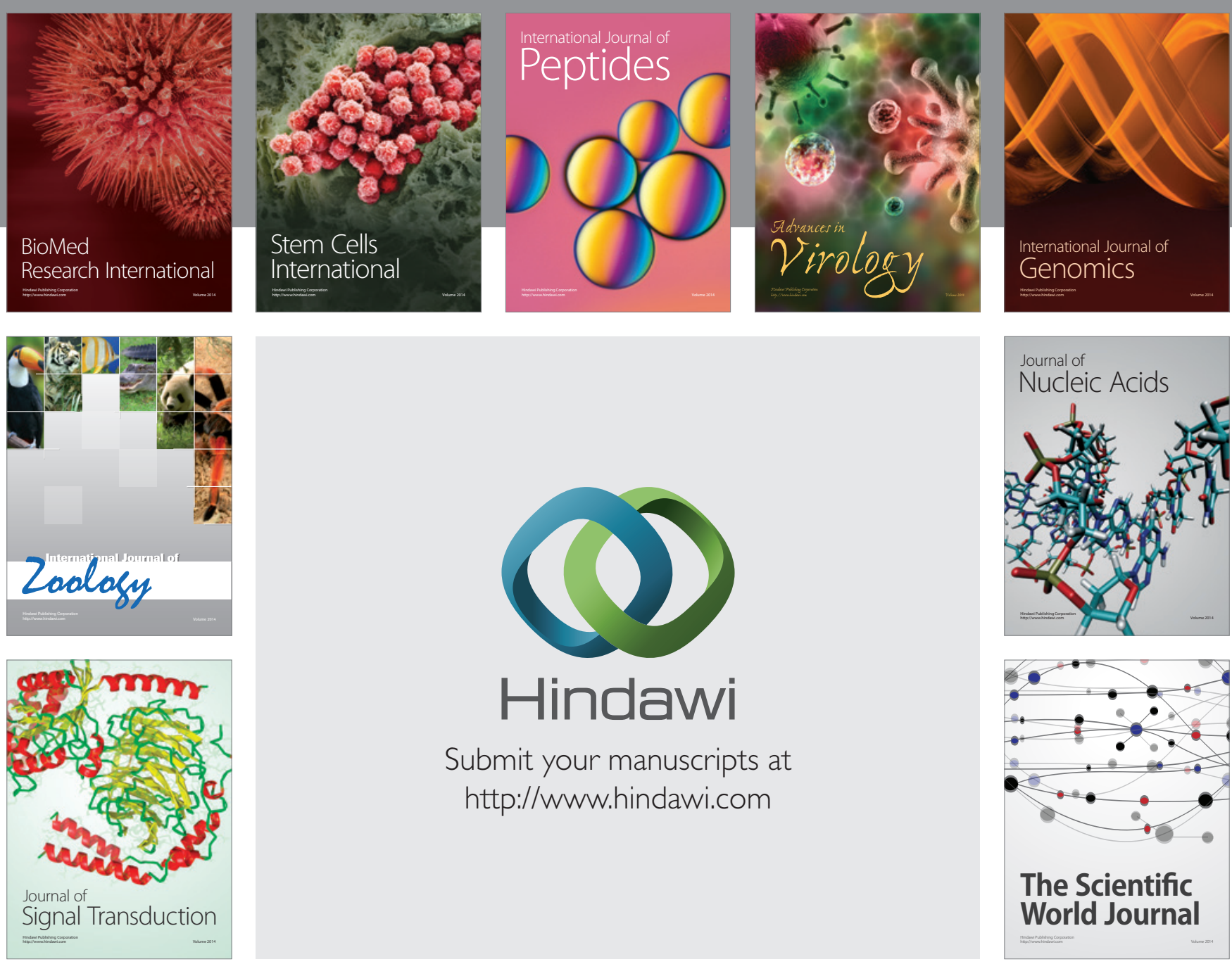

Submit your manuscripts at

http://www.hindawi.com
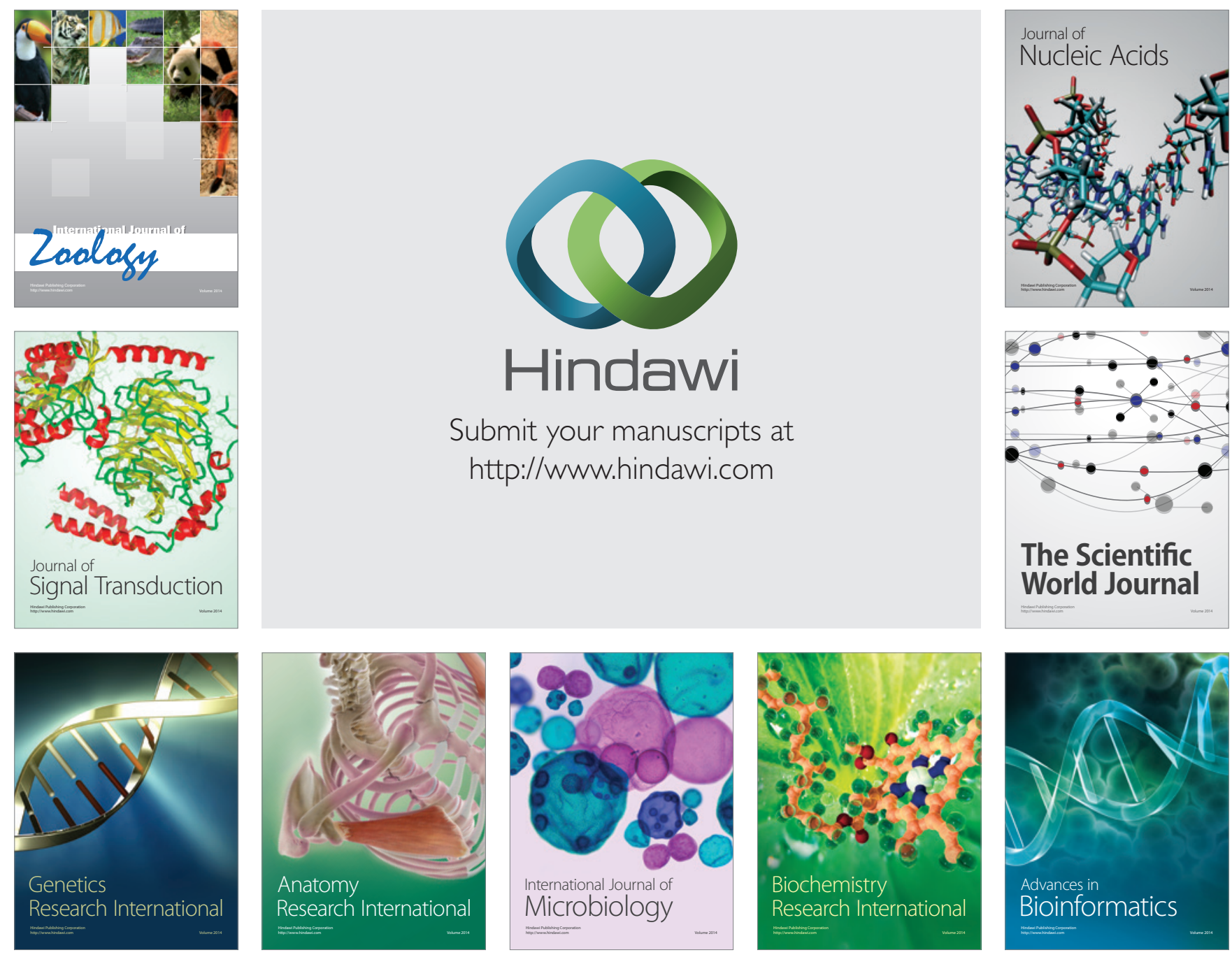

The Scientific World Journal
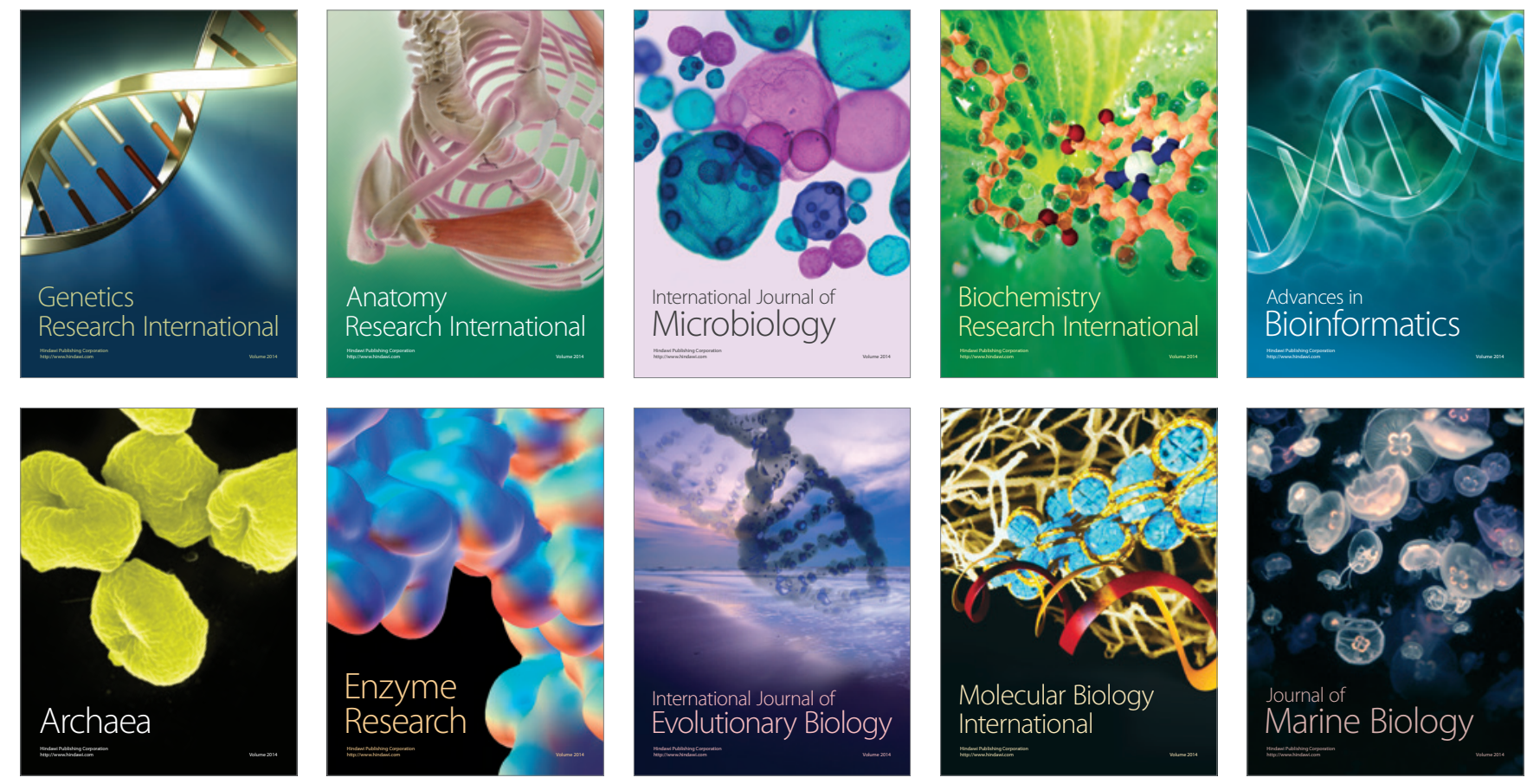